\title{
How Associative Thinking Influences Scene Perception
}

\author{
Shira Baror ${ }^{1,2}$, Moshe Bar ${ }^{1}$, Elissa Aminoff ${ }^{3}$ \\ ${ }^{1}$ The Gonda Multidisciplinary Brain Research Center, Bar Ilan University, Israel \\ ${ }^{2}$ Neuroscience Institute, New York University School of Medicine, New York, USA \\ ${ }^{3}$ Department of Psychology, Fordham University, Bronx, USA
}

Corresponding Author:

Elissa M. Aminoff, Ph.D.

Department of Psychology

Fordham University

441 E. Fordham Rd

Bronx, NY 10458

eaminoff@fordham.edu

718-817-3480 


\begin{abstract}
Perception of our external environment is not isolated from the influence of our internal thoughts. Here, we investigate the nature of their interaction. We argue that a common associative mechanism underlies both the perception of scenes and our internal thoughts, and in three experiments hypothesize that there is a functional advantage to an associative thought pattern in the perception of scenes. Experiments 1 and 2 showed that associative thinking indeed facilitates scene perception, an effect that evolved over the course of the experiments. Experiment 3 showed that associative thinking hinders the perception of individual, isolated objects, in which associative information is minimized, but that object perception is facilitated when associative thinking is reduced. This double dissociation reveals that a match between the orientation of internal and external processing is key for perception, suggesting that an associative mind is more receptive of externally perceived associative information.
\end{abstract}

Keywords: Scene perception, Associations, State of Mind, Mindsets, Thought, Default Mode Network 


\section{Introduction}

Almost every human experience interweaves internal thought and perception of the external environment. For example, imagine sitting on the beach and enjoying the sunset. Your eyes appreciate the visual scene while your mind wanders in thought. How do these thoughts influence your perception of the scene in front of you? This question has been tackled from various directions, testifying to its complexity, and its centrality to cognitive science. Nevertheless, we still don't have a comprehensive framework for how thought interacts with perception.

Some theories suggest that thoughts and perception compete for attention, such that mindsets that require more internal focus come at the expense of processing perceptual inputs, resulting, for example, in enhanced inattentional blindness ${ }^{1}$ or in perceptual decoupling ${ }^{2,3}$. Other lines of research contend that thought may serve as a top-down cue for perception by priming and ultimately facilitating a perception of a stimulus to match and correspond with what is internally represented $^{4,5}$ (though see [6]). Beyond theories that predominantly focus on attentional competition or content-level priming, recent theories suggest that thought and perception may be interdependent based on a shared form or orientation of processing. For example, the Construal Level Theory predicts that abstract levels of thinking are linked with global perceptual processing ${ }^{7}$. Similarly, the Overarching States of Mind framework ${ }^{8}$ postulates that different elements encompassing one's state of mind (i.e., perception, attention, thought, openness to experience and affect) are interdependent, and that the processing mode in one element (e.g., thought) influences processing of other elements (e.g., perception).

In line with these theories, here we bring scene perception and thought-related processing together and hypothesize that an associative mode of thought influences scene perception due to shared fundamental processing, elaborated below. 
Natural scene perception requires the integration of individual elements ${ }^{9}$, as well as the extraction of global gist ${ }^{10,11}$, and it involves the processing of spatial information ${ }^{12}$, textures ${ }^{13}$ and viewpoints ${ }^{14}$. Most notably, scene perception relies on processing contextual associations of various kinds, learned over multiple co-occurrences. Much of the information we process in a scene relates to object-to-object associations and their relations within their spatial setting ${ }^{15}$. Demonstrating this effect, activity in scene-preferring brain regions was modulated by the contextual associations afforded by the visual stimulus ${ }^{16,17}$. Moreover, in exploring an associative account for scene processing, Aminoff and colleagues demonstrated that the mere acquiring of associations, even in the absence of semantic meaning, engaged scene-selective brain regions ${ }^{18,19}$. This evidence-based account highlights the rich associative nature of scenes.

Associativity in the external processing of scenes may be compatible with associativity in the internal realm of our state of mind ${ }^{8}$ - our fluctuating stream of thought ${ }^{20}$. States of mind, or mindsets, have specifically been delineated in terms of the associative processes they involve. For example, a creative state of mind is suggested to rely on processing associations in a novel yet useful way ${ }^{21}$. Abilities such as associative flexibility (i.e., diversity in associations) and associative fluency (i.e., number of associations) are found to represent a core function underlying creativity $^{22,23}$. Additionally, remoteness of associations is a strong indicator of divergent thinking, a primary measurement of creative thought ${ }^{24}$. Spontaneous thought as well, and the state of 'mind wandering', was also suggested to be sustained by associative processing ${ }^{25,26}$. More globally, Christoff et al., (2016) ${ }^{20}$ define spontaneous thought by aligning different mindsets such as creativity, mind wandering, ruminations and sleep, along an axis indicating the level of associativity and variability each of them involves. 
Associative perception and associative mindset have been mostly studied under two different umbrellas; the former focusing on visual tasks and the latter focusing on mental, and often memory-related processing. Nonetheless, we hypothesized that compatibility in associative processing is an organizing principle governing the interaction between mindset and perception. Specifically, we hypothesize that an associative mindset will modulate scene perception. This hypothesis is indirectly supported by data showing that spontaneously evoked associations taking place during memory encoding ${ }^{27}$, or self-referential episodic thought ${ }^{28,29}$ activate scene-selective brain regions. Nevertheless, a direct examination of the hypothesized interaction between the two in behavior has yet to be explored. We examine this hypothesis by testing scene perception via a 1-back scene similarity task while promoting mindset in different levels of associativity, thus, manipulating associative processes in internal thoughts and in external perception. Perceptual performance here relies on processing the unique associations each scene triggers, especially when different scenes belong to the same category. Using this paradigm, we found that an associative mindset facilitates scene perception.

It should be noted, that while in natural settings thought and perception take place simultaneously, in most lab settings they have been manipulated in a serial manner (with thought usually priming perception) and assessed in different time scales (with associative thought probed over the order of seconds to minutes and associative perceptual processing in the order of milliseconds). Here, we designed a novel paradigm to interrogate the parallel processing of both perception and thought by having participants perform a perceptual task while artificially inducing the associative degree of their mindset. With this paradigm, performance on the scene perception task can be assessed in light of the degree to which the mindset was orientated towards associative processing. Furthermore, the time course of a perception-thought interaction could evolve in 
several ways: for example, it could be slow with the mindset manipulation becoming more influential over time (e.g., resulting from growing proficiency in the task), or it could be fast and become less influential over time (e.g., leading to fatigue). The potential interdependent influence could also remain at a fixed level throughout the experiment. In order to address this open question, we divided the experiments into three consecutive blocks. Previewing the results, in all three experiments we found that the interaction of an associative mindset with associative visual perception evolved over time, with maximum enhancement of an associative mindset on associative scene perception found at the last block of each experiment.

\section{Experiment 1}

Experiment 1 was aimed to examine how different mindsets influence scene perception. To that aim, scene images were presented consecutively, and participants were asked to respond to each image and judge whether it was identical or different from the preceding image. Simultaneously, words that elicited different mindsets were superimposed on the presented images, and participants were asked to remember them for a subsequent memory test. If mindset interact with perception, we hypothesized that the varying mindset conditions would lead to performance changes in the scene perception task.

\section{Methods}

\section{Stimuli}

Stimuli were scene images used for the perceptual task, and word lists used for the mindset manipulation. The scene images for the perceptual task were chosen from the BOLD5000 dataset $^{30}$. This large dataset includes 5000 images as well as the fMRI BOLD signal results of four participants viewing these images. Images were $375 \times 375$ pixels and subtended approximately 9.5 
degrees of visual angle. For the purpose of our studies, we used a sub-sample of this dataset, which includes 250 scene categories, each having four exemplar images. Of these four images, we chose the two images among the four exemplars, that showed the highest correlation in BOLD signal in the parahippocampal place area (PPA) and retrosplenial complex (RSC), the core brain regions suggested to sustain scene perception through contextual associative processing ${ }^{16}$. While these image pairs belonged to the same category (e.g., a playground), they did not necessarily share all their comprising features, such as their viewpoint or their comprising elements. This selection process resulted in a total of 500 images ( 250 categories with two exemplars each) that were subsequently embedded in the task. Examples for such associative-based scene pairs are depicted in Fig 1a. 
a
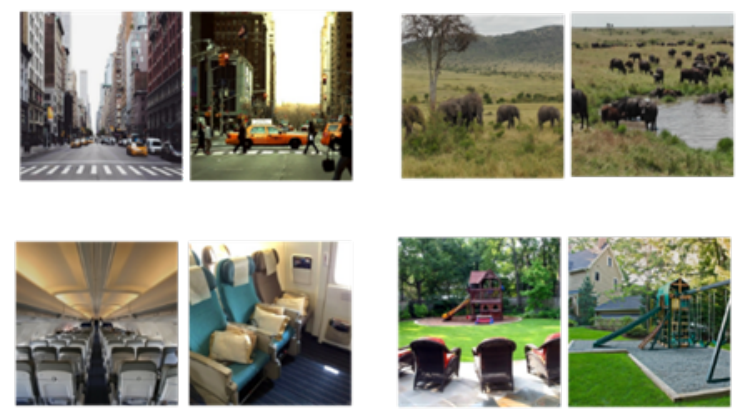

b

\begin{tabular}{ccccc} 
‘Narrow' list & & 'Broad' list & & 'Unrelated' list \\
\cline { 1 - 1 } Teeth & & Strawberry & & Violin \\
Mouth & & Banana & & Ring \\
White & & Monkey & & Ham \\
Gums & & Cage & & Earrings \\
Toothbrush & & Bird & Rabbit \\
Toothpaste & & Bird feeder & Egg \\
Dentist & & Seeds & Piano \\
Braces & & Bagel & Ears \\
Brush & Cream cheese & Tuxedo \\
Chew & Salmon & Easter \\
Smile & & River & Omelet \\
Floss & & Raft & Wedding
\end{tabular}

C

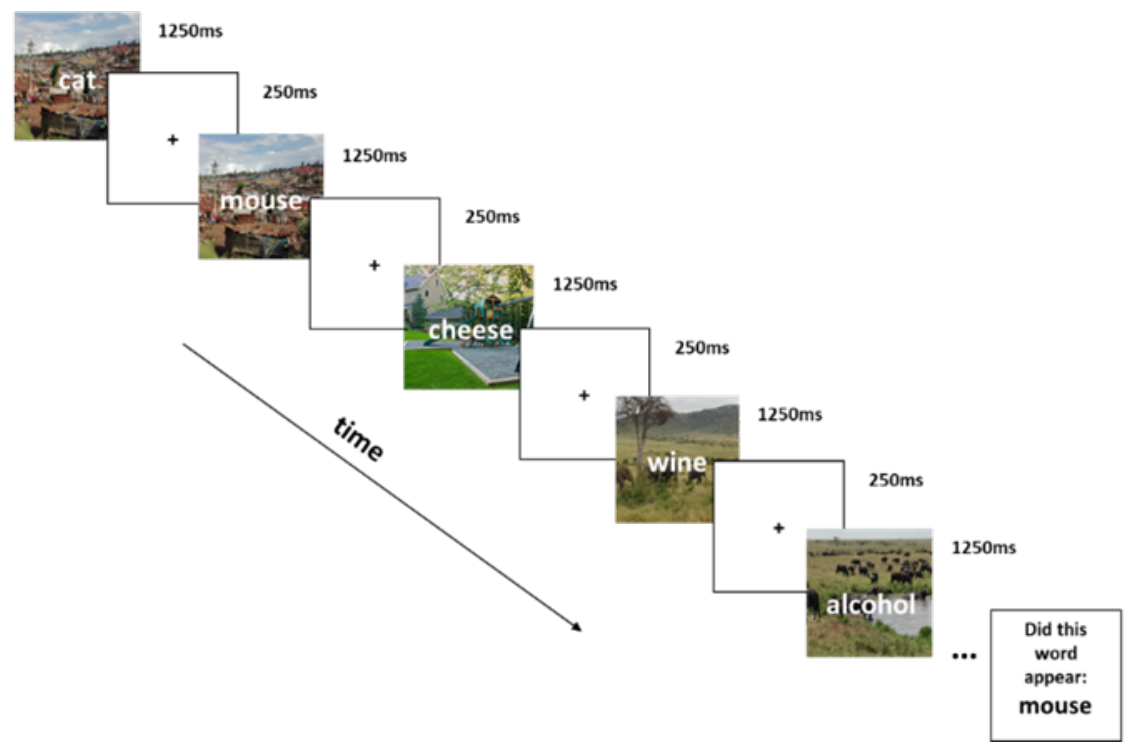

Figure 1: Stimuli and experimental design of Exp. 1. a, Examples of scene stimuli. The image stimuli consisted of scenes from the BOLD5000 data $\operatorname{set}^{30}$. The examples depict pairs of scene exemplars that share a category but are not identical. b, Mindset examples. The stimuli consisted of lists of twelve words each. In the Narrow condition all words are associatively related to the same context. In the Broad condition, only consecutive words are associatively related to the same context, but the list as a whole raises multiple unrelated contexts. In the Unrelated condition, words do not share a context at all. c, An array example. Words were superimposed on the images, both presented for $1250 \mathrm{~ms}$ in each trial, and followed by a $250 \mathrm{~ms}$ presentation of a cross. Following twelve trials, participants were shown one word and were asked to determine whether that word appeared as part of the array or not. The example depicts a list from the Broad condition.

Word lists for the mindset manipulation were adapted from Mason \& Bar $(2012)^{31}$ and comprised three levels of associativity. In the 'narrow' condition, words were all contextually 
associated to one another, all focusing on one, narrow contextual theme (e.g., dog-cat-puppyanimal). In this case there would be rich associative processing of the context. In the 'broad' condition, each word was associated to the word preceding it and to the word following it, but the list as a whole involved a broad rather than a narrow set of contexts, presumably eliciting more dispersed associative processing (e.g., tomato-red-blood-knife). In the 'unrelated' condition, words were completely unrelated to one another, such that associative processing was minimized (e.g., whistle-helmet-shovel-mitten). Each list comprised 12 words, and each mindset condition comprised a total of 24 lists. An example list for each mindset condition is depicted in Fig $1 \mathrm{~b}$.

\section{Design}

The experiments involved a 1-back scene similarity task. Images of scenes were presented one after the other, interleaved with fixation crosses, and participants were asked to determine as fast as possible with regards to every image whether it was identical to or different from the previously presented image. The task comprised three image conditions: images could be identical to the previous image (i.e., 'same' condition), completely different from the previous image (i.e., 'different' condition) or similar in their contextual category to the image preceding them yet different in the specific details and layout (i.e., 'similar' condition).

Simultaneously to the perceptual scene task, participants' mindsets were manipulated by the presentation of the word lists. These word lists were successfully used in the past to affect one's mindset ${ }^{31}$. Words in each list were sequentially presented, superimposed on top of the scene images at screen center. Participants were asked to attend to the presented words in parallel to actively reporting their perceptual same/different decisions regarding the scene images. To verify attention to the words, participants were asked to remember these words for a following memory recognition test at the end of the array. In each thirteenth trial, a word was presented against a 
white background, and participants were asked to determine whether that word appeared in the previous sequence or not. In $50 \%$ of the cases the test word appeared as part of the sequence and in $50 \%$ of the cases the test word was novel yet associated with one of the words in the sequence.

To summarize the conditions in the paradigm- the perceptual task comprised three image conditions, such that images could be either the same as, similar to, or completely different from their preceding image. The memory-words task comprised three mindset conditions, which were either narrowly associated with one another, broadly associated, or unrelated.

Each trial included a presentation of a scene image, and a presentation of a word superimposed on the image at screen center. The content of the words in each list did not overlap the content of the images. Every image-word pair was presented for the duration of $1250 \mathrm{~ms}$, and was followed by a $250 \mathrm{~ms}$ presentation of a fixation cross. Presentation duration was not influenced by response reaction time (RT). Trials were grouped into arrays of twelve, such that the presentation of each 12 words-list was paired with and superimposed over the presentation of 12 consecutive scene images.

Scene images were randomized across lists. Additionally, image conditions were randomized within each list, such that in each 12-trials array, 50\% were images identical to their preceding image (i.e., 'same condition'), and 50\% were of images different from their preceding image. Of the different images, 50\% were images completely different from the preceding image (i.e., 'different condition') and 50\% were different from the preceding image in their details yet similar to the preceding image in their category (i.e., 'similar condition'). This division of trials was made to make sure the responses are not biased to making a 'same' or 'different' response in any of the arrays. An example of several consecutive trials is depicted in Fig. 1c. 
The experiment comprised three blocks (i.e., beginning, middle, and end), and each block contained a total of 24 lists, eight consecutive lists from each mindset condition, counterbalanced in their order across blocks. This arrangement was aimed to verify that the narrow, broad and unrelated mindset conditions all appeared at the beginning (first block), middle (second block) and end (third block) of the experiment, such that possible differences between mindset conditions cannot be accounted for by practice or time into the experiment. Breaks were introduced between each 8 consecutive 12-trials arrays that belonged to the same mindset conditions. This was meant to allow rest periods throughout the experiment.

\section{Procedure}

Upon arriving to the experiment, participants signed informed consent forms and were administered a short practice stage that included a total of 12 lists, 4 from each mindset condition, each list counterbalancing all scene image conditions within it. Participants received feedback on their memory performance during practice regarding their memory of the test word at the end of every list. After completing the practice stage and verifying that they understood the task, the participants completed the full experiment. The entire experimental process took $\sim 50$ minutes, after which participants were thanked and compensated, either with money or with course credits. This experiment, as well as Experiments 2 and 3 were run in a quiet room. Participants were seated in front of the monitor, with their head positioned at $\sim 60 \mathrm{~cm}$ from the center of the screen. All experiments were run on iMac using MATLAB psychtoolbox. All three experiments reported here were approved by Fordham University's Institutional Review Board. Informed consent was obtained from all participants. All research was performed in accordance with the Declaration of Helsinki.

\section{Results}


35 participants took part in the experiment ( 22 females, mean age $=21.13$ years). Four participants were excluded from analysis due to poor performance in the scene perception task (below 2.5 std from the mean). Two participants were excluded from analysis due to poor performance in the memory task (below 2.5 std from the mean). One participant was excluded due to not performing the task correctly (responded only to images that repeated themselves). All further analysis was performed based on the data of the remaining 28 participants.

In analyzing the results, we first looked at the memory performance to make sure that participants attended the words, and that their attention did not vary across mindset conditions. We then examined performance in the scene perception task, analyzing all three image conditions (i.e., same, similar, different) in both accuracy and in RT, under the varying mindset conditions (i.e., narrow, broad, unrelated). We hypothesized that mindset differences in associative processing will influence scene perception, operationalized as how they performed in the 1-back task. Lastly, to evaluate the time course related to the effectiveness of the mindset manipulation, we were interested in how performance varied as a function of the duration into the experiment (first, middle and end block), introducing this variable as another focus of analysis.

Memory analysis did not yield accuracy differences between the mindset conditions, thus verifying that attention to the words did not vary across mindset conditions. Memory means for the narrow, broad and unrelated mindset conditions were: $0.78,0.76,0.77$, respectively $(\mathrm{F}(26,2)=$ $0.4, \mathrm{p}<0.6)$. Nonetheless, a significant interaction between mindset and order of blocks emerged $(F(24,4)=2.79, p<0.03$, observed power $=0.71)$. Post-hoc analysis shows that this interaction stems from accuracy decline over time in the broad mindset condition $(F(26,2)=6.31, p<0.006$, observed power= 0.86$)$. 
RT analysis of memory performance reveals a significant main effect for mindset condition, such that $\mathrm{RT}$ in the unrelated condition $(\mathrm{M}=907 \mathrm{~ms}, \mathrm{SD}=34 \mathrm{~ms})$ was slower than $\mathrm{RT}$ in the narrow $(\mathrm{M}=870 \mathrm{~ms}, \mathrm{SD}=26 \mathrm{~ms})$ and the broad $(\mathrm{M}=871 \mathrm{~ms}, \mathrm{SD}=29 \mathrm{~ms})$ conditions $(\mathrm{F}(26,2)=$ $5.58, \mathrm{p}<0.01$, observed power $=0.81)$.

Next, we turn to analyze performance of the scene perception task. This analysis includes only trials in which memory of the words was correct.

Accuracy analysis of the scene perception task, which focused on hit rate, revealed a main effect for image condition, such that accuracy in the task was the highest when images were completely different from the image preceding them $(\mathrm{M}=0.95, \mathrm{SD}=0.006)$, followed by images that were identical to the image preceding them $(\mathrm{M}=0.93, \mathrm{SD}=0.007)$. Lowest accuracy was found for images that were similar in their category yet different in their details from the image preceding them $(\mathrm{M}=0.91, \mathrm{SD}=0.009 ; \mathrm{F}(26,2)=26.8, \mathrm{p}<0.0009$, observed power $=1)$.

Accuracy analysis also showed a triple interaction between the image condition, the mindset condition and the order of blocks $(\mathrm{F}(20,8)=5.42, \mathrm{p}<0.001$, observed power $=0.98)$. This interaction revealed that in the similar image condition, which involved sensitivity to the scene's associations to the greatest extent, accuracy rate increased over time under the narrow mindset, and deteriorated under the unrelated mindset (Fig. 2a). Subsequent pairwise comparison analyses revealed that under the unrelated mindset, performance in block 3 was significantly worse from performance in block 1 ( $<<0.003$; corrected for multiple comparisons).

RT analysis of the scene perception task revealed a complementary result. First, a significant effect of image condition was found, such that RT in the similar image condition $(\mathrm{M}=$ $742 \mathrm{~ms}, \mathrm{SD}=24 \mathrm{~ms})$ was significantly slower than $\mathrm{RT}$ in the same $(\mathrm{M}=696 \mathrm{~ms}, \mathrm{SD}=21 \mathrm{~ms})$ and 
different $(M=704 \mathrm{~ms}, \mathrm{SD}=25 \mathrm{~ms})$ conditions $(\mathrm{F}(26,2)=51.51, \mathrm{p}<0.0009$, observed power=1). Secondly, a significant effect of mindset condition was found for the scene task, such that RT was significantly faster in the narrow $(M=708 \mathrm{~ms}, \mathrm{SD}=22 \mathrm{~ms})$ and broad $(\mathrm{M}=696 \mathrm{~ms}, \mathrm{SD}=22 \mathrm{~ms})$ mindset, compared with the unrelated mindset $(\mathrm{M}=738 \mathrm{~ms}, \mathrm{SD}=25 \mathrm{~ms} ; \mathrm{F}(26,2)=12.85, \mathrm{p}<0.0009$, observed power=0.99).

Most importantly a triple interaction emerged between the image conditions, the mindset conditions and the order of blocks $(\mathrm{F}(20,8)=2.22, \mathrm{p}<0.03$, observed power $=0.83)$. This interaction demonstrated a double dissociation. Over time, RT was significantly faster under the narrow mindset and significantly slower under the unrelated mindset. No significant over-time RT changes were observed in the broad mindset. Post-hoc analysis revealed that this interaction was significant in the similar and in the different image conditions (Fig. 2b; Graphs are presented for the similar image condition, in which the results were most evident, but see Table 1 for the complete set of statistical information from all conditions in all experiments).

Subsequent pairwise comparison analyses revealed that under the narrow mindset, in the similar image condition, performance in block 3 was significantly faster compared with block 1 $(p<0.002)$ and block $2(p<0.0009)$. In the different image condition, faster performance was found in block 1 compared with block $3(\mathrm{p}<0.02)$. Under the unrelated mindset, in the similar image condition, significant differences were found between all blocks (block1-2: $\mathrm{p}<0.0009$, block 2-3: $\mathrm{p}<0.004$, block 3-1: $\mathrm{p}<0.01$ ) such that performance was slowest in block 2 and fastest in block 1 . In the different image condition performance in block 2 was the slowest and was significantly different from block $1(\mathrm{p}<0.01)$ and block $3(\mathrm{p}<0.01)$. All analyses were corrected for multiple comparisons. 


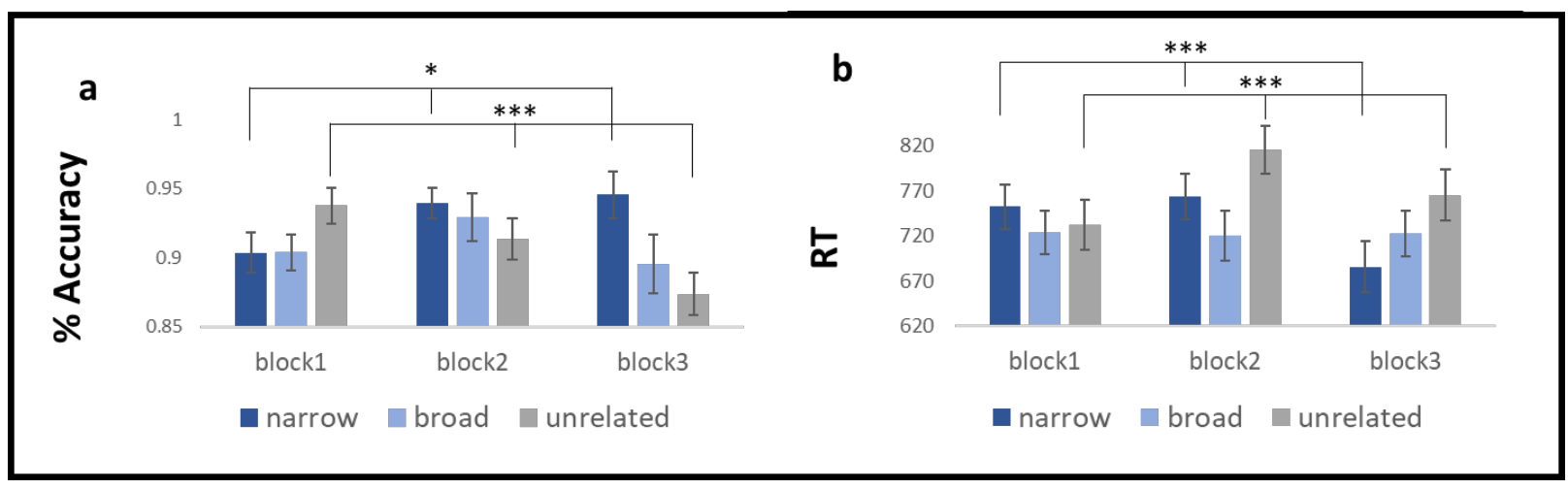

Figure 2: Results of the 'similar' image condition in Exp. 1. a, Accuracy. b, RT. Figures depict the interaction between mindset conditions (i.e., narrow, broad and unrelated) and the order of blocks (first to last). Bars indicate standard error of the mean. Significant main effects are denoted by asterisks.

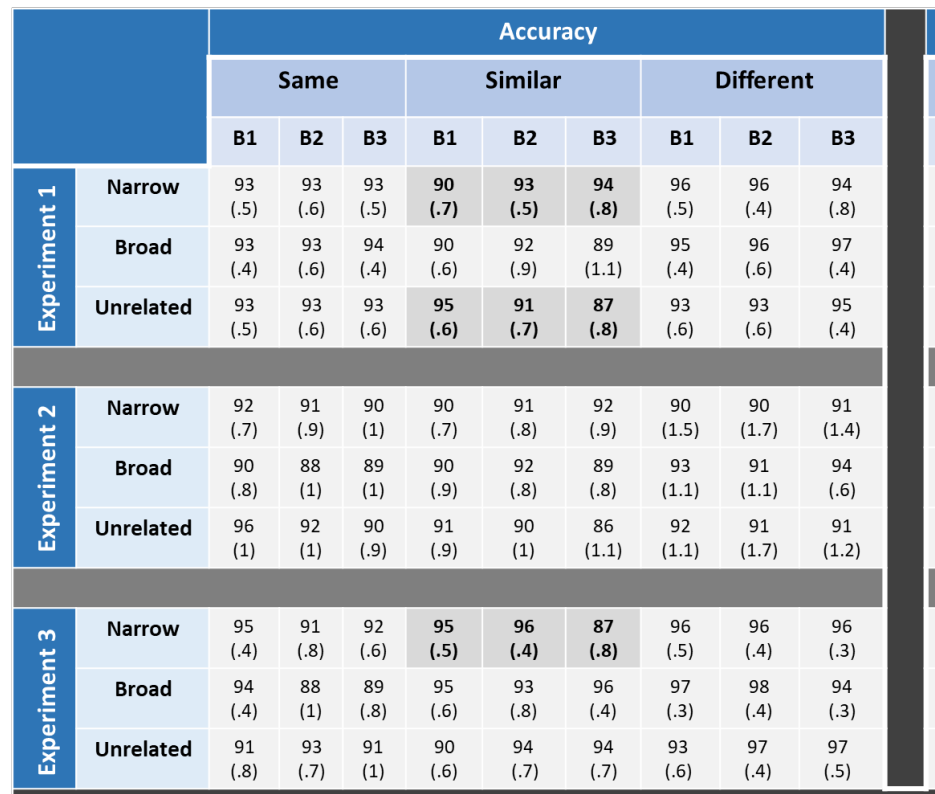

\begin{tabular}{|c|c|c|c|c|c|c|c|c|}
\hline \multicolumn{9}{|c|}{ RT } \\
\hline \multicolumn{3}{|c|}{ Same } & \multicolumn{3}{|c|}{ Similar } & \multicolumn{3}{|c|}{ Different } \\
\hline B1 & B2 & B3 & B1 & B2 & B3 & B1 & B2 & B3 \\
\hline $\begin{array}{c}696 \\
(108)\end{array}$ & $\begin{array}{c}713 \\
(134)\end{array}$ & $\begin{array}{c}663 \\
(109)\end{array}$ & $\begin{array}{c}752 \\
(130)\end{array}$ & $\begin{array}{c}763 \\
\text { (131) }\end{array}$ & $\begin{array}{c}685 \\
(149)\end{array}$ & $\begin{array}{c}721 \\
\text { (127) }\end{array}$ & $\begin{array}{c}702 \\
\text { (159) }\end{array}$ & $\begin{array}{c}677 \\
(136)\end{array}$ \\
\hline $\begin{array}{c}672 \\
(113)\end{array}$ & $\begin{array}{c}680 \\
(126)\end{array}$ & $\begin{array}{c}689 \\
(136)\end{array}$ & $\begin{array}{c}723 \\
(126)\end{array}$ & $\begin{array}{c}719 \\
(147)\end{array}$ & $\begin{array}{c}722 \\
(134)\end{array}$ & $\begin{array}{c}676 \\
(136)\end{array}$ & $\begin{array}{c}693 \\
(162)\end{array}$ & $\begin{array}{c}687 \\
(136)\end{array}$ \\
\hline $\begin{array}{c}693 \\
(130)\end{array}$ & $\begin{array}{c}736 \\
(128)\end{array}$ & $\begin{array}{c}720 \\
(138)\end{array}$ & $\begin{array}{c}731 \\
(146)\end{array}$ & $\begin{array}{c}815 \\
(140)\end{array}$ & $\begin{array}{c}764 \\
(151)\end{array}$ & $\begin{array}{c}701 \\
(160)\end{array}$ & $\begin{array}{c}761 \\
(150)\end{array}$ & $\begin{array}{c}717 \\
(157)\end{array}$ \\
\hline $\begin{array}{l}643 \\
(91)\end{array}$ & $\begin{array}{l}641 \\
(95)\end{array}$ & $\begin{array}{c}634 \\
(107)\end{array}$ & $\begin{array}{c}765 \\
(114)\end{array}$ & $\begin{array}{c}739 \\
(118)\end{array}$ & $\begin{array}{c}696 \\
(137)\end{array}$ & $\begin{array}{c}716 \\
(121)\end{array}$ & $\begin{array}{c}693 \\
(123)\end{array}$ & $\begin{array}{c}671 \\
(119)\end{array}$ \\
\hline $\begin{array}{c}646 \\
(115)\end{array}$ & $\begin{array}{l}626 \\
(94)\end{array}$ & $\begin{array}{c}652 \\
(106)\end{array}$ & $\begin{array}{c}720 \\
(139)\end{array}$ & $\begin{array}{c}714 \\
(132)\end{array}$ & $\begin{array}{c}734 \\
(135)\end{array}$ & $\begin{array}{c}692 \\
(130)\end{array}$ & $\begin{array}{c}679 \\
(130)\end{array}$ & $\begin{array}{c}694 \\
(139)\end{array}$ \\
\hline $\begin{array}{c}638 \\
(106)\end{array}$ & $\begin{array}{l}646 \\
(84)\end{array}$ & $\begin{array}{l}644 \\
(89)\end{array}$ & $\begin{array}{c}723 \\
(125)\end{array}$ & $\begin{array}{c}773 \\
(104)\end{array}$ & $\begin{array}{c}735 \\
\text { (111) }\end{array}$ & $\begin{array}{c}691 \\
(141)\end{array}$ & $\begin{array}{c}717 \\
(106)\end{array}$ & $\begin{array}{c}712 \\
(127)\end{array}$ \\
\hline $\begin{array}{c}660 \\
(117)\end{array}$ & $\begin{array}{c}652 \\
(109)\end{array}$ & $\begin{array}{l}655 \\
(81)\end{array}$ & $\begin{array}{c}696 \\
(121)\end{array}$ & $\begin{array}{l}679 \\
(85)\end{array}$ & $\begin{array}{l}751 \\
(96)\end{array}$ & $\begin{array}{c}650 \\
(123)\end{array}$ & $\begin{array}{l}674 \\
(85)\end{array}$ & $\begin{array}{c}739 \\
(115)\end{array}$ \\
\hline $\begin{array}{l}658 \\
(93)\end{array}$ & $\begin{array}{c}665 \\
(120)\end{array}$ & $\begin{array}{c}649 \\
(100)\end{array}$ & $\begin{array}{c}709 \\
(115)\end{array}$ & $\begin{array}{l}736 \\
(83)\end{array}$ & $\begin{array}{c}664 \\
(69)\end{array}$ & $\begin{array}{c}691 \\
(107)\end{array}$ & $\begin{array}{c}693 \\
(108)\end{array}$ & $\begin{array}{l}649 \\
(49)\end{array}$ \\
\hline $\begin{array}{c}677 \\
(111)\end{array}$ & $\begin{array}{c}650 \\
(114)\end{array}$ & $\begin{array}{l}660 \\
(85)\end{array}$ & $\begin{array}{l}745 \\
\text { (91) }\end{array}$ & $\begin{array}{c}689 \\
(127)\end{array}$ & $\begin{array}{l}680 \\
(98)\end{array}$ & $\begin{array}{c}732 \\
(104)\end{array}$ & $\begin{array}{l}638 \\
\text { (95) }\end{array}$ & $\begin{array}{l}669 \\
(89)\end{array}$ \\
\hline
\end{tabular}

Table 1: Complete statistical descriptives for the scene task. The table shows the mean and the std (in parenthesis) in all experiments (Exp. 1, Exp. 2, Exp. 3), in both accuracy (left) and RT (in ms, right) measures, as they change as a function of image conditions (same, similar or different) and across experimental blocks (B1, B2, B3). Highlighted are slots in which the main effect of change across the experimental blocks was statistically significant. 
These results demonstrate that scene processing occurs faster and more accurately when performed under a mindset in which associative processing is augmented, compared with under a mindset in which associative processing is reduced. These findings are most apparent when processing consecutive scene images that are different exemplars of the same scene category.

\section{Experiment 2}

Exp. 1 incorporated a memory recognition test to verify attention to the presented words simultaneously to performing the perceptual task. While this procedure was aimed to motivate attention to the words, to indeed trigger the different mindset conditions, it is possible that remembering the words was not equally demanding across conditions, and the results in Exp. 1 are confounded by variations in memory load. To tease apart mindset associativity from memory load, Exp. 2 was conducted in which words were presented but the memory task was eliminated. This allowed us to examine whether the mere exposure to concepts that vary in their encompassed associative information is enough to influence perception.

\section{Methods}

All stimuli in Exp. 2 were identical to the stimuli used in Exp. 1. The only difference between the experiments is that in Exp. 2, participants were not explicitly asked to attend to the presented words, as task performance was measured with regards to the scene images, but not with regards to the word lists. Here, participants were told that the words appearing superimposed on top of the images are not the focus of their task, and that they can choose to ignore them. Instead of a memory recognition test, following every 12-trials array a white screen appeared for three seconds, and participants were encouraged to let their eyes rest from the perceptual scene task 
during this short intermission. As there was no memory task in Exp. 2, participants did not receive feedback on their performance during practice.

\section{Results}

26 participants took part in the experiment ( 15 females, mean age $=19.38$ years $)$. Two participants were excluded from analysis due to poor performance in the scene perception task (below 2.5 std from the mean), therefore all further analysis was performed based on the data of the remaining 24 participants.

Accuracy analysis of the scene perception task did not reveal a significant effect of the image conditions nor did it reveal a significant effect of the mindset conditions. Nonetheless, RT analysis revealed significant effects that resemble the results in Exp. 1. First, a significant effect of image condition was found, such that all image conditions were significantly different from one another. Slowest RT's were found in the similar image condition $(\mathrm{M}=733 \mathrm{~ms}, \mathrm{SD}=23 \mathrm{~ms})$, followed by the different image condition $(\mathrm{M}=696 \mathrm{~ms}, \mathrm{SD}=23 \mathrm{~ms})$. Fastest $\mathrm{RT}$ was found in the same image condition $(\mathrm{M}=641 \mathrm{~ms}, \mathrm{SD}=18 \mathrm{~ms} ; \mathrm{F}(22,2)=67.21, \mathrm{p}<0.0009$, observed power=1).

Most importantly a triple interaction emerged between the image conditions, the mindset conditions and the order of blocks $(\mathrm{F}(16,8)=2.76, \mathrm{p}<0.04$, observed power $=0.77)$. This interaction shows a double dissociation similar to that found in Exp. 1. Over time, RT was significantly faster under the narrow mindset, unchanged in the broad condition, and significantly slower under the unrelated mindset. As in Exp. 1, this was most apparent in the similar image condition, in which fine sensitivity to scene's associative content is most required (Fig. 3). Subsequent pairwise comparison analyses revealed that under the narrow mindset, in the similar image condition, performance in block 3 was significantly faster compared with block $1(\mathrm{p}<0.001)$ 
and block $2(\mathrm{p}<0.01)$. In the different image conditions, the significant difference was found between block 3 and block $1(\mathrm{p}<0.03)$. Under the unrelated mindset, in the similar image condition, performance in block 1 was significantly faster than performance in block $2(\mathrm{p}<0.03)$. All analyses were corrected for multiple comparisons.

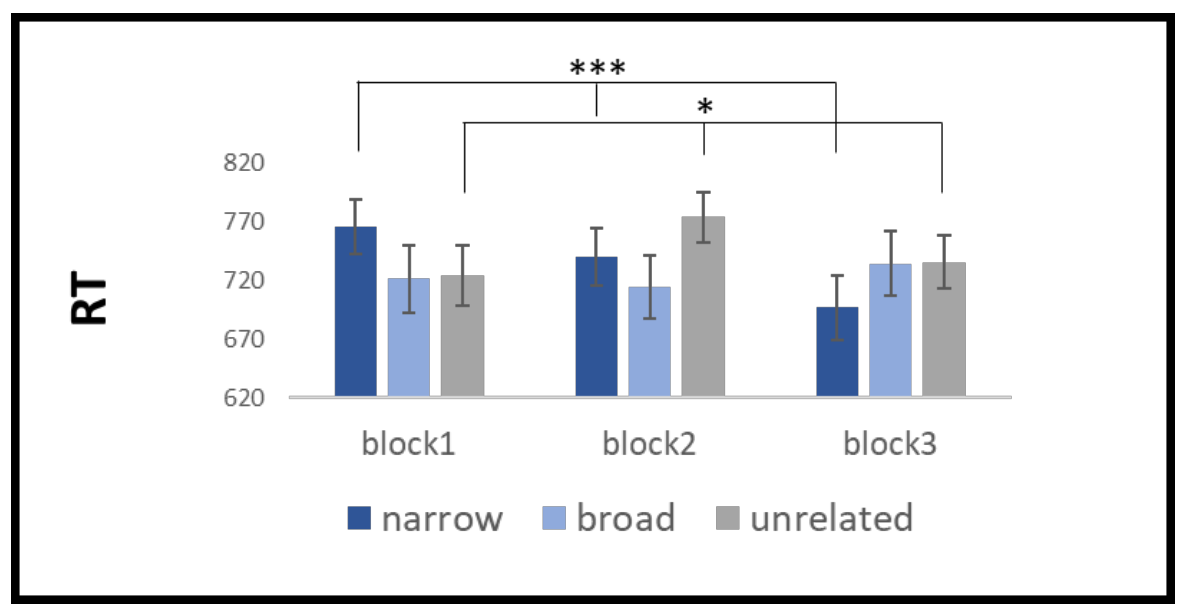

Figure 3: RT results of 'similar' image condition in Exp. 2. Figure depicts the interaction between mindset conditions (i.e., narrow, broad and unrelated) and the order of blocks (first to last). Bars indicate standard error of the mean. Significant main effects are denoted by asterisks.

Exp. 2 replicates the facilitatory effect of an associative mindset on scene processing, and is not confounded by memory load.

\section{Experiment 3}

Exp. $1 \& 2$ revealed that an associative mindset facilitates scene perception, but it is possible that an associative mindset facilitates any perceptual task, regardless of the involved associative affordance of the visual stimuli. To dissociate a general enhancement effect from an associative-related effect, we conducted Exp. 3 which involved images of mundane individual objects that are similar to scenes in their naturalness, yet minimize associative processing. If an associative mindset selectively enhances associative-based perception because of the shared 
associative orientation, then it will not enhance perception of objects with minimal associations. Alternatively, if an associative mindset has a global facilitatory effect on perception, then object perception will be enhanced.

\section{Methods}

\section{Stimuli}

The main goal of experiment 3 was to evaluate whether the influence of the associative mindset on perceptual processing is specific to scenes, or whether it enhances perception of any stimuli. We therefore used daily objects as stimuli, images that are of items as meaningful and natural as scenes, but that have been contrasted with scenes in the past in terms of recognition processes and associative involvement. The object images for the perceptual task were chosen from a dataset by Brady et al., (2008) ${ }^{32}$. This dataset includes images of various objects, each having more than one exemplar. Because this experiment was aimed to examine the influence of mindset on perceptual performance in which associative processing is minimized, we chose 265 objects that are not associated with a specific context (e.g., a t-shirt rather than a wedding tuxedo). The objects chosen were inspired by the original set of weak contextual objects defined in Bar \& Aminoff $(2003)^{16}$. For each object we chose two different exemplars. This selection resulted in a total of 530 images to be used in the experiment. Examples for these object image pairs are depicted in Fig. 4a. The word lists for the mindset manipulation were identical to the ones used in Exp. 1 and Exp. 2. 
a
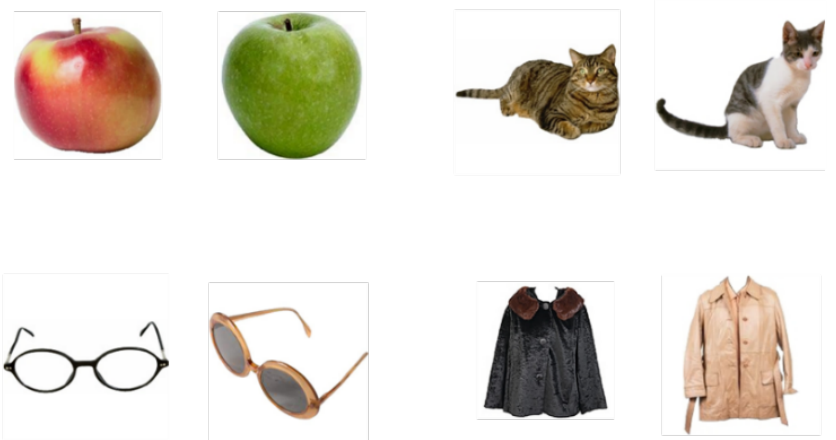

b

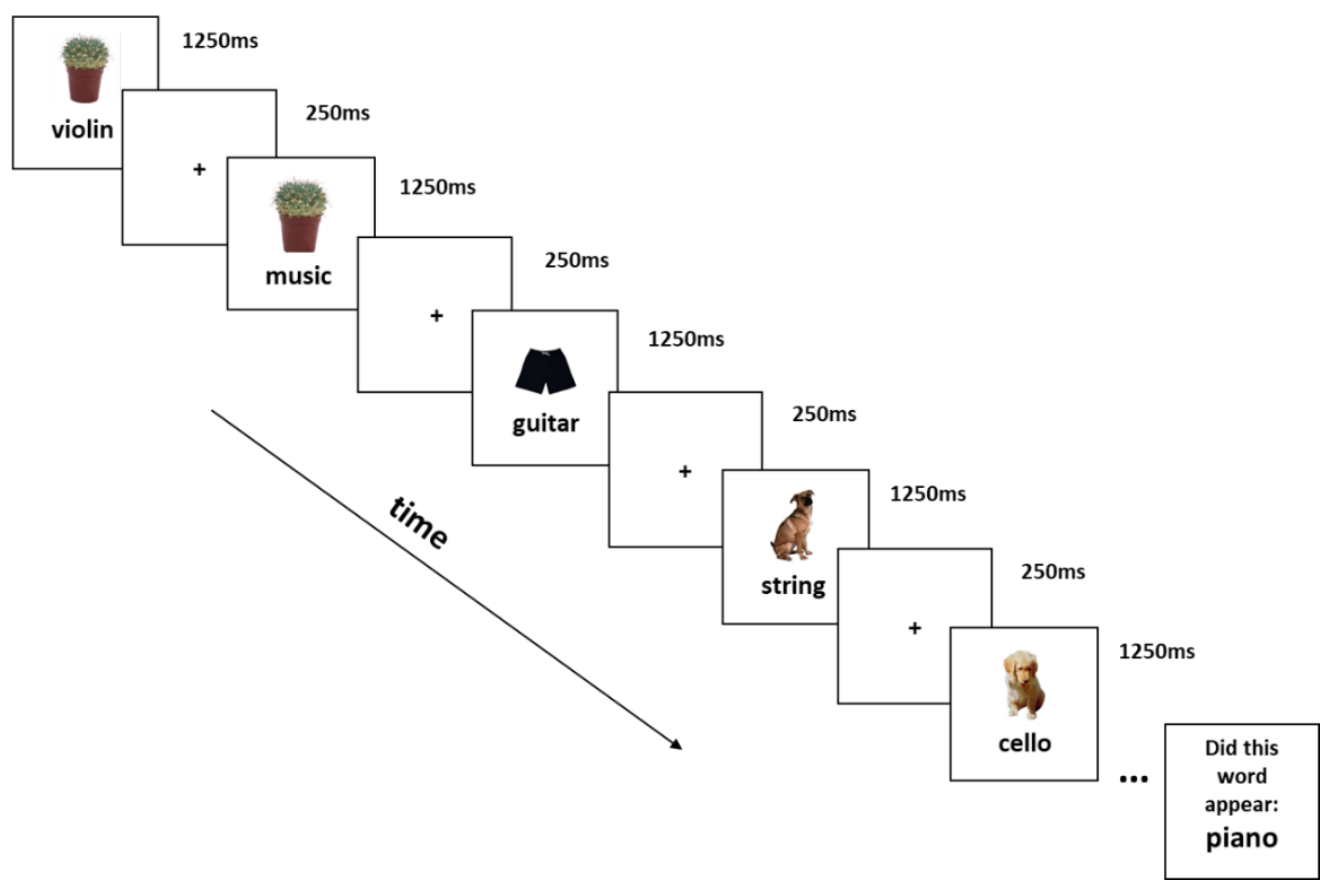

Figure 4: Stimuli and experimental design of Exp. 3. a, Stimuli examples. The image stimuli consisted of mundane objects. The examples depict pairs of object exemplars which share a category but do not trigger a specific context. b, An array example. Words were presented below the images, and both were presented for $1250 \mathrm{~ms}$ in each trial, and followed by a $250 \mathrm{~ms}$ presentation of a cross. As in Exp. 1, following twelve trials, participants were shown one word and were asked to determine whether that word appeared as part of the array or not. 


\section{Design}

In Exp. 3, a 1-back task was employed in which images of objects were presented one after the other (interleaved with fixation crosses) and participants were asked to determine as fast as possible with regards to every image whether it is identical to or different from the previously presented image. The task comprised three image conditions, such that images could be either identical to the previous image (i.e., same condition), completely different from the previous image (i.e., different condition) or a different exemplar of the image preceding them (i.e., similar condition). This was aimed to conduct the task in the most similar manner to the first two experiments, yet dissociate between experiments in the affordance of associative information.

In addition to the perceptual object task, participants' mindset was manipulated by the presentation of the word lists that were used in Exp. 1 and Exp. 2. Words in each list were sequentially presented, such that they appeared simultaneously and below the object images (see Fig. 4b). Participants were asked to attend the presented words in parallel to actively reporting their perceptual same/different decisions regarding the object images. As in Exp. 1, participants were asked to remember the words for a following memory recognition test. Similarly to Exp. 1, the experiment's design involved three conditions in the object perception task (i.e., same, similar and different) and three mindset conditions (i.e., associative, intermediate and unrelated). This experiment was identical to Exp. 1 in its design and procedure, with the exception that the images were objects, and the words were presented below the image.

\section{Results}

13 participants took part in the experiment $(12$ females, mean age $=19.46)$. This relatively small sample size is due to the outbreak of COVID-19 pandemic, which did not allow running further 
experimental sessions. One participant was excluded from analysis due to poor performance in the object perception task (below 2.5 std from the mean), therefore all further analysis was performed based on the data of the remaining 12 participants.

Memory analysis did not yield accuracy differences between the mindset conditions $(\mathrm{F}(2,10)=2.04, \mathrm{p}<0.1)$, nor did it yield RT differences $(\mathrm{F}(2,10)=1.03, \mathrm{p}<0.2)$.

Accuracy analysis of the object perception task revealed a main effect for images, such that accuracy in the task was the highest when images were completely different from the image preceding them $(\mathrm{M}=0.96, \mathrm{SD}=0.008)$, compared with images in the same $(\mathrm{M}=0.92, \mathrm{SD}=0.01)$ and similar image conditions $(\mathrm{M}=0.93, \mathrm{SD}=0.01 ; \mathrm{F}(10,2)=16, \mathrm{p}<0.0009$, observed power= 0.99). This result replicates the result from Exp. 1.

Additionally, an interaction emerged between the mindset conditions and the order of blocks $(F(8,4)=3.6, p<0.03$, observed power $=0.66)$. This interaction shows the opposite of the findings in Exp. 1 and 2. Specifically, in the similar image condition accuracy increased over time under the unrelated mindset, and decreased under the narrow associative mindset (Fig. 5a). Subsequent pairwise comparison revealed that under the narrow mindset, in the similar image condition a significant difference is found between block 2 and block 3, where performance was worse in block 3 compared with block 2 ( $<<0.05$, corrected for multiple comparisons).

RT analysis of the object perception task revealed a complementary result. First, a significant effect of image condition was found, such that RT in the similar image condition $(\mathrm{M}=$ $706 \mathrm{~ms}, \mathrm{SD}=24 \mathrm{~ms})$ was significantly slower than $\mathrm{RT}$ in the same $(\mathrm{M}=659 \mathrm{~ms}, \mathrm{SD}=26 \mathrm{~ms})$ and different $(\mathrm{M}=682 \mathrm{~ms}, \mathrm{SD}=23 \mathrm{~ms})$ image conditions $(\mathrm{F}(10,2)=11.71, \mathrm{p}<0.002$, observed power= $0.96)$. 
Additionally, an interaction emerged between the image conditions, the mindset conditions and the order of blocks $(F(8,4)=3.36, p<0.01$, observed power $=0.83)$. This interaction showed a double dissociation in an opposite trend from Exp. 1 and 2. Over time, RT was faster under the unrelated mindset, and slower under the associative mindset. As in Exp. 1, post-hoc analysis reveals that this interaction was significant in the different and in the similar image condition (Fig. $5 b)$. Subsequent pairwise comparison analyses revealed that under the narrow mindset, in the similar image condition, performance was significantly slower in block 3 compared with block 2 $(p<0.001)$. In the different image conditions, performance in block 3 was significantly slower than block $1(\mathrm{p}<0.04)$ and $2(\mathrm{p}<0.04)$. Under the unrelated mindset however, in the similar image condition, performance in block 1 was significantly slower than performance in block $2(\mathrm{p}<0.01)$ and $3(\mathrm{p}<0.008)$. Similarly, in the different image conditions, performance in block 1 was significantly slower than block $2(p<0.002)$ and $3(p<0.02)$. All analyses were corrected for multiple comparisons.

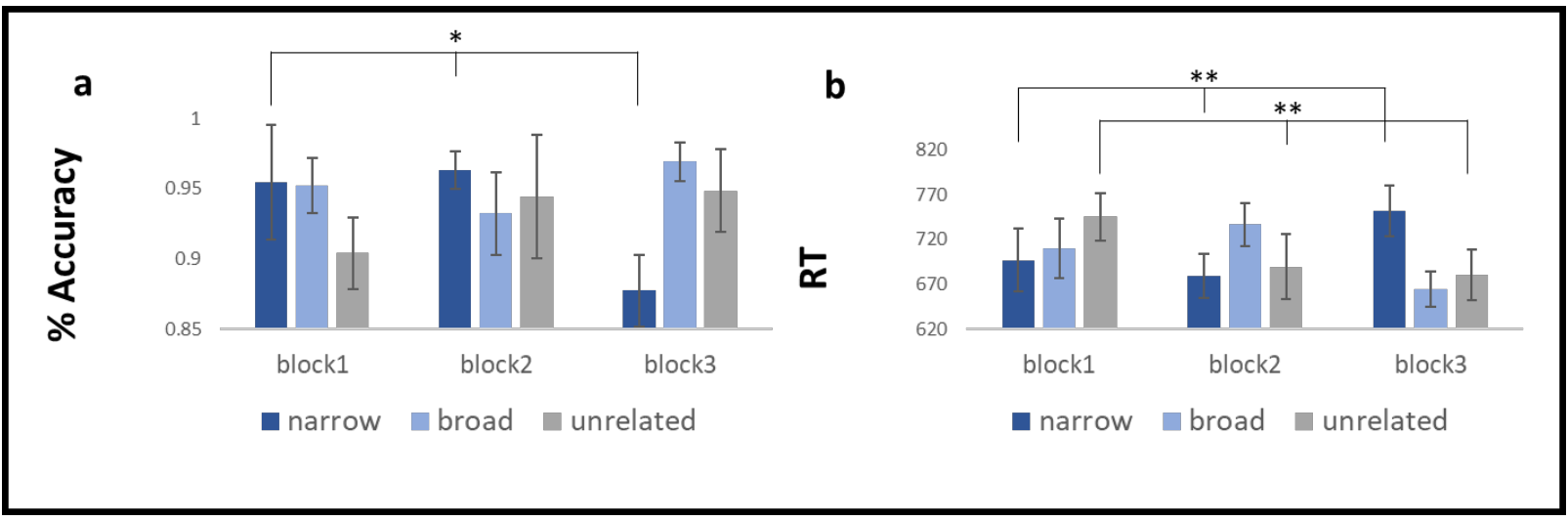

Figure 5: Results of 'similar' image condition in Exp. 3. a, Accuracy. b, RT. Figures depict the interaction between mindset conditions (i.e., narrow, broad, and unrelated) and the order of blocks (first to last). Bars indicate standard error of the mean. Significant main effects are denoted by asterisks. 
Exp. 3 shows that the positive effect of an associative mindset on perception depends on the associative affordance of the given perceptual process. Furthermore, when taking together the findings in all experiments, a double dissociation between the form of thought and the form of perception is revealed. Whereas scene perception is facilitated under an associative mindset (and hindered under an un-associative one), perception of objects with minimal associations benefit from a mindset that does not involve associative processing (and hindered by an associative one).

\section{Follow-up analysis}

All three experiments revealed an interaction between mindset and perceptual processing. To better evaluate whether these interactions are equivalent across experiments, we conducted a follow-up planned comparisons analysis which focused on RT difference in performance between the first and third block in every experiment.

For each participant, we subtracted RT in the first block from RT in the third block for each the image and mindset conditions. A planned-comparison mixed ANOVA revealed a significant interaction between the influence of mindset on perceptual processing and the experiment $(\mathrm{F}(122,4)=8.43, \mathrm{p}<0.0009$, observed power $=0.99)$, showing that while in Exp. 1 and 2 scene perception was enhanced over time under the narrow mindset and deteriorated under the unrelated mindset, in Exp. 3 object perception deteriorated under the narrow mindset and improved under the unrelated mindset (Fig. 6).

An additional triple interaction emerged, between the experiments, the mindset condition and the image condition $(F(116,8)=4.3, p<0.0009$, observed power $=0.97)$. This interaction shows that the most significant difference between experiments stems from the narrow associative mindset and the similar image condition which involves associative processing to the greatest 
extent. This finding implies that the most significant difference between the experiments that involved scene perception (i.e., Exp. 1 and 2) and the experiment involving object perception (i.e., Exp. 3) was indeed the associative processing.

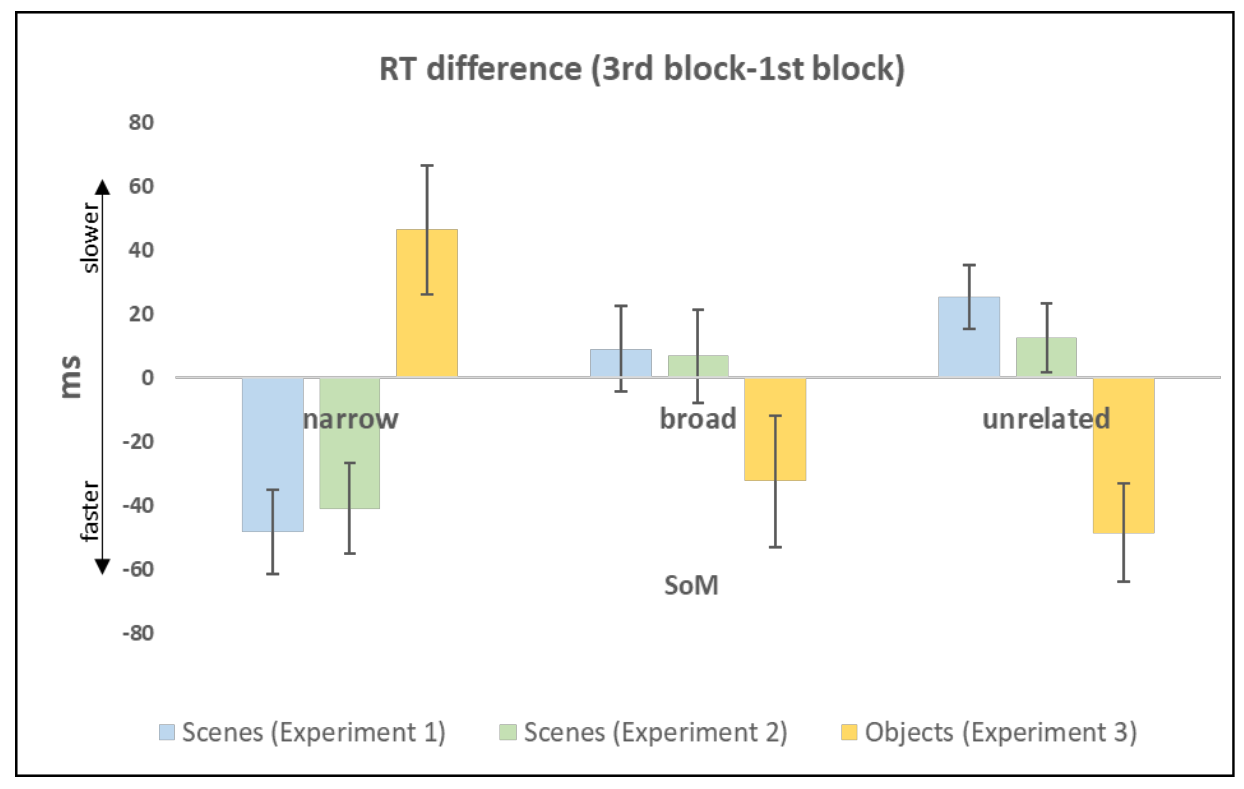

Figure 6: Follow up analysis. Comparing Exp. 1,2,3 by the subtracting RT in the first block from RT in the third block in each experiment. A positive difference in ms depicts slower performance over time and a negative difference depict faster performance over time. An interaction is found between experiments and mindset conditions. Under the narrow mindset, Scene perception (Exp. 1 and 2) is facilitated and object perception (Exp. 3) is delayed. Complementarily, under the unrelated mindset scene perception is delayed and object perception is facilitated $(\mathrm{F}(122,4)=8.43, \mathrm{p}<0.0009)$. Bars indicate standard error of the mean. Significant differences are denoted by asterisks. The figure is collapsed across scene conditions.

\section{Discussion}

Associative processes have been studied in the context of scene perception as well as in the context of different modes of thought. Nonetheless, despite being intertwined in everyday life, the possible interaction between associative processing in thought and perception has rarely been studied. Addressing this gap, we explored whether scene perception is influenced by an associative mindset. 
Exp. 1 showed that over time, an associative mindset facilitates scene perception. Exp. 2 replicated this result, and further excluded a memory-load based explanation. Critically, Exp. 3 showed that visual processing of isolated objects that do not elicit many contextual associations is hindered by an associative mindset, showing that an associative mindset selectively enhances associative-based scene perception.

The influence of mindset on perception has been demonstrated in the past, predominantly explained by means of priming. Previous research showed that the content of thoughts, memories and goals influence conscious perception ${ }^{4,5,33}$ and perceptual decision-making regarding related content $^{34}$. These studies suggest that thought influences perception by providing a priming simulation of the content to be subsequently perceived ${ }^{35}$. Here, however, the content triggered by the mindset manipulation was not related to the content of the simultaneously presented scenes. Therefore content-related mechanisms such as priming or spreading activation cannot explain our findings. Instead, independent from priming of content, our findings suggest that the form of thought facilitates the form of perceptual processing; a cross-domain priming of the 'how' rather than the 'what'. This proposal pertains to the benefit in the match between the associative way in which thought and perception are structured. This is demonstrated in Exp. 1 \& Exp. 2, in which participants were more receptive of scene-related information when thought was associatively organized, and is further corroborated in Exp. 3, where participants were more receptive of unassociative perceptual information when thought was organized in an un-associative manner. This shows that perception is not isolated from thought, but can be more efficient and receptive when processing is matched, or impaired when the underlying processes are different.

Interestingly, in all three studies, we did not find an influence of the manipulated 'broad' condition on scene perception. This condition is complex because it involved strong associative 
processing between consecutive pairs of words at the local level, but at the global, list-level, it elicited multiple unrelated contexts instead of a rich unified context, as in the narrow condition. We believe when all words of the list were related to one another, as in the narrow condition, this elicited a rich associative context critical to creating an associative mindset. However, in the broad condition, this combination of elements - strongly associative locally (in the consecutive pairs) but globally (list-level) unassociated - could have facilitated scene perception since associative processing was required to identify the associations between consecutive words. However, the associative integrity may have not reached a threshold, as it does in the narrow condition with the rich associations, needed to affect perceptual processing. Alternatively, scene perception could have been hampered under this condition if one was more attentive to the global unrelatedness, and by so perhaps even inhibiting local associative processing of the words. It is therefore not unlikely that under the setting of the current experiments, strong local associativity embedded within global unrelatedness led to a cancellation effect in this condition. Future studies that specifically probe one's attention in this complex condition may shed more light on the magnitude of associative processing involved.

Which neural systems sustain the brain's associative mode? A good candidate is activity in the Default Mode Network (DMN), a neural network that underlies associative, spontaneous cognition ${ }^{19,20,36,37}$. The DMN overlaps with the Contextual Associations Network, which was found to uniquely underlie the processing of visual contextual associations ${ }^{38,39}$, most predominantly found in conjunction with scene processing ${ }^{16}$. This set of brain regions, which is referred to as the DMN in the thought literature, and as the Contextual Associations Network in the perception literature, has been suggested to centralize around a shared, domain-independent function, of proactively processing associative information ${ }^{19,40}$. Our studies provide empirical 
evidence for such a domain-general associative mechanism, and it is possible that an associative mindset, which activates the DMN, interacts with scene processing, which elicits associations of the perceptual input.

If this interpretation is correct, then our findings elucidate ongoing discussions regarding the relationship between the DMN and task-related processing. Some accounts have previously conceptualized the DMN's functional significance as sustaining task-unrelated thought, building upon observed DMN deactivation when engaged in demanding perceptual tasks ${ }^{41}$. Nevertheless, most of these visual tasks did not involve associative processing ${ }^{19}$. Therefore, rather than exemplifying a tension between external and internal processes, these accounts may have been confounded by differences in associative affordance, namely, involving rich associative processes in the thought domain, and minimizing associative processes in the perceptual domain. Here we show that associative thought, possibly driven by DMN activity, facilitates rather than contradicts task-related perceptual performance when both processes are compatible in terms of their associative processing mode, supporting DMN accounts that highlight its primary role in processing associations, independent from how they are triggered ${ }^{42}$.

Taking our studies together, some open questions still remain. First, the stimuli were presented for long durations $(>1000 \mathrm{~ms})$. How much was this a factor in yielding the results remains unknown. Does extensive processing of a scene need to occur to see an effect of associative thought, or is the initial rapid processing of a scene vulnerable to the effects of interdependence in mindset? Future studies (e.g., using EEG) should investigate whether the effect we found is occurring at early phases of scene processing ( 200ms, e.g., [43]); or affecting later processing stages of scene processing (e.g., [44]). In addition, future studies should parametrically adjust stimulus presentation duration to see if extensive viewing of a scene is required for the effect 
to occur. Associative processing of objects was found to occur as early as $150 \mathrm{~ms}^{45}$ and thus, we may predict the effect of this interdependence to modulate an initial, early - stage process of scene perception. However associative processing has shown to modulate scene processing in later stages as well ${ }^{46}$ and the interdependence of the mindset effect may be salient at late processing as well. Secondly, with regards to the third experiment, beyond manipulating objects instead of scenes, this experiment was not identical to the first two experiments in that the words were presented below the object image rather than superimposed on it, therefore we cannot rule out the possibility that the opposite pattern of findings observed in the third experiment may have also resulted from this difference in design. While we cannot fully address these caveats in the current design, we propose that both of these points can be addressed by implementing eye-tracking and neuroimaging methods to similar designs in the future. Tracking one's eye movements during scene or object perception while their mindset is manipulated may shed light, both on the trajectory of attention to associations between elements in the scene, as well as to the attentional division between the words and the images.

Another key question that remains open is the one of differences in temporal dynamics between the thought and perception domains. While perceptual and mindset-related associative processes are suggested to engage a shared mechanism, the time-course of their engagement may nonetheless be different from one another. Scene perception relies on immediate associations that are triggered by the stimulus, at a timescale of milliseconds ${ }^{43}$. Associative processing in thought, however, is assumed to result from the 'mental train of thought'; the representation of consecutive associated events ${ }^{47}$, which dictates a slower temporal course of engagement unfolding over at least several seconds ${ }^{48}$. Our results exemplify this temporal distinction, as the enhancement of scene processing by an associative mindset evolved over the course of the experiments. Further research 
is required to understand how the gradually evolving mental associative process informs the immediate perceptual one. One method for mitigating this challenge in temporal discrepancies is using electroencephalogram (EEG) recordings which permits both the observation of rapid event related potentials (ERP) as well as measuring oscillations over longer timeframes. EEG studies show that scene perception is tied with the P200 ERP component ${ }^{43}$ and that changes in alpha power recorded over seconds, are implicated with spontaneous thought $t^{47}$ and with DMN activity ${ }^{50}$. It is possible that changes in alpha oscillations that result from inhabiting an associative mindset, provide an internal neural state which then shapes the more local P200 scene-related ERP. Similarly, conducting fMRI studies in mixed block/event related designs ${ }^{51,52}$ may provide another effective tool for jointly studying transient changes in associative based scene perception and sustained activity related to associative mindset.

\section{Concluding remarks}

Almost every human experience involves both thought and perception, yet the nature of their interaction is poorly understood. Our studies show that perceptual task performance is enhanced when one's thought-related mode of processing is in accordance. In specific, we found that the associative affordance of the perceptual process at hand was facilitated with an associative mode of thought, and point in the direction of a domain-general associative mechanism, possibly sustained by DMN activity. Our work calls for a paradigm shift, in which different cognitive and perceptual functions are studied under a unified framework, similarly to how they are intertwined in our undivided brain. 


\section{References}

1. Shi, Y., \& Li, H. How a crisis mindset activates intuitive decision process: role of inattentional blindness. Psychological Research, 85(2), 592-604. (2020).

2. Schooler, J.W., Smallwood, J., Christoff, K., Handy, T.C., Reichle, E.D., \& Sayette, M.A. Meta-awareness, perceptual decoupling and the wandering mind. Trends in Cognitive Sciences, 15, 319-326. (2011).

3. Smallwood, J., Brown, K. S., Tipper, C., Giesbrecht, B., Franklin, M. S., Mrazek, M. D., \& Schooer, J. W. Pupillometric evidence for the decoupling of attention from perceptual input during offline though. PLoS ONE, 6, e18298. (2011).

4. Scorolli, C., Borghi, A. M., \& Glenberg, A. Language-induced motor activity in bi-manual object lifting. Experimental Brain Research, 193, 43-53. (2009).

5. Brunyé, T. T., Walters, E. K., Ditman, T., Gagnon, S. A., Mahoney, C. R., \& Taylor, H. A. The fabric of thought: priming tactile properties during reading influences direct tactile perception. Cognitive Science, 36(8), 1449-1467. (2012).

6. Firestone, C., \& Scholl, B. J. Cognition does not affect perception: Evaluating the evidence for top-down effects. Behavioral and Brain Sciences, 39. (2016).

7. Trope, Y., \& Liberman, N. Construal-level theory of psychological distance. Psychological Review, 117(2), 440-463. (2010).

8. Herz, N., Baror, S., \& Bar, M. Overarching states of mind. Trends in Cognitive Sciences, 24, 184-199. (2020).

9. Kim, J. G., \& Biederman, I. Where do objects become scenes? Cerebral Cortex, 21, 17381746. (2011).

10. Bar, M. Visual objects in context. Nature Review Neuroscience 5:617-629. (2004). 
11. Torralba, A., Oliva, A., Castelhano, M. S., \& Henderson, J. M. Contextual guidance of eye movements and attention in real-world scenes: The role of global features in object search. Psychological Review, 113, 766-786. (2006).

12. Epstein, R.A., \& Kanwisher, N. A cortical representation of the local visual environment. Nature, 392(6676), 598-601. (1998).

13. Park, J., \& Park, S. Conjoint representation of texture ensemble and location in the parahippocampal place area. Journal of Neurophysiology, 117, 1595-1607. (2017).

14. Park, S., \& Chun, M. M. Different roles of the parahippocampal place area (PPA) and retrosplenial cortex (RSC) in scene. NeuroImage, 47(4), 1747-1756. (2009).

15. Biederman, I., Mezzanotte, R.J., \& Rabinowitz, J.C. Scene perception: Detecting and judging objects undergoing relation violations. Cognitive Psychology, 14: 143-177. (1982).

16. Bar, M., \& Aminoff, E. M. Cortical analysis of visual context. Neuron, 38, 347-358. (2003).

17. Bar, M., Aminoff, E.M, \& Schacter, D.L. Scenes unseen: The parahippocampal cortex intrinsically subserves contextual associations, not scenes or places per se. Journal of Neuroscience, 28(34), 8539-8544. (2008).

18. Aminoff, E. \& Tarr, M. Associative processing is inherent in scene perception. PLoS One 10:e128840. (2015).

19. Aminoff, E., Gronau, N. \& Bar, M. The parahippocampal cortex mediates spatial and nonspatial associations. Cerebral Cortex 17(7):1493-503. (2007).

20. Christoff, K., Irving, Z.C., Fox, K.C.R., Spreng, R.N., \& Andrews-Hanna, J.R. Mindwandering as spontaneous thought: A dynamic framework. Nature Reviews Neuroscience, 
17, $718-731$. (2016).

21. Mednick, S.A. The associative basis of the creative process. Psychological Review, 69, 220-232. (1962).

22. Benedek, M., Könen, T., \& Neubauer, A.C. Associative abilities underlying creativity. Psychology of Aesthetics, Creativity, and the Arts, 6, 273-281. (2012).

23. Beaty, R.E., Silvia, P.J, Nusbaum, E.C., Jauk, E. \& Benedek, M. The role of associative and executive processes in creative cognition. Memory \& Cognition, 42, 1186-1197. (2014).

24. Acar, S., \& Runco, M.A. Assessing associative distance among ideas elicited by tests of divergent thinking. Creativity Research Journal, 26 (2014), 229-238. (2014).

25. Andreasen, N.C., O’Leary, D.S., Cizadlo, T., Arndt, S., Rezai, K., Watkins, G.L., Boles Ponto, L.L., \& Hichwa, R.D. Remembering the past: two facets of episodic memory explored with positron emission tomography. American Journal of Psychiatry, 152:15761585. (1995).

26. Bar, M., Aminoff, E., Mason, M., \& Fenske, M. The units of thought. Hippocampus, 17(6), 420-428. (2007).

27. Peters, J., Daum, I., Gizewski, E., Forsting, M. \& Suchan, B. Associations evoked during memory encoding recruit the context-network. Hippocampus, 19, 141-151. (2009).

28. Szpunar, K.K., Chan, J.C., \& McDermott, K.B. Contextual processing in episodic future thought. Cerebral Cortex, 19:1539-48. (2009).

29. Gilmore, A.W., Nelson, S.M., \& McDermott, K.B. The contextual association network activates more for remembered than for imagined events. Cerebral Cortex, 26:611-617. (2016). 
30. Chang, N., Pyles, J.A., Marcus, A., Gupta, A., Tarr, M.J., \& Aminoff, E.M. BOLD5000, a public fMRI dataset while viewing 5000 visual images. Scientific Data, 6, 49. (2019).

31. Mason, M.F., \& Bar, M. The effect of mental progression on mood. Journal of Experimental Psychology, 141(2), 217-221. (2012).

32. Brady, T.F., Konkle, T., Alvarez, G.A. \& Oliva, A. Visual long-term memory has a massive storage capacity for object details. Proceedings of the National Academy of Sciences, USA, 105 (38), 14325-14329. (2008).

33. Radel, R. \& Clément-Guillotin, C. Evidence of motivational influences in early visual perception: Hunger modulates conscious access. Psychological Science, 23:232-34. (2012).

34. Bartholow, B. D., \& Heinz, A. Alcohol and aggression without consumption: Alcohol cues, aggressive thoughts, and hostile perception bias. Psychological Science, 17, 30-37. (2006).

35. Hesslow, G. Conscious thought as simulation of behavior and perception. Trends in Cognitive Sciences, 6, 242-247. (2002).

36. Mason, M. F., Norton, M. I., Horn, J. D. Van, Wegner, D. M., Grafton, S. T., \& Macrae, C. N. Wandering minds: Stimulus-independent thought. Science, 315, 393-395. (2007).

37. Raichle, M.E., MacLeod, A.M., Snyder, A.Z., Powers, W.J., Gusnard, D.A., \& Shulman, G.L. A default mode of brain function. Proceedings of the National Academy of Sciences of the United States of America, 98(2), 676-682. (2001).

38. Diana, R.A., Yonelinas, A.P., \& Ranganath, C. Adaptation to cognitive context and item information in the medial temporal lobes. Neuropsychologia 50:3062-3069. (2012).

39. Rauchs, G., Orban, P., Balteau, E., Schmidt, C., Degueldre, C., Luxen, A., Maquet, P., \& Peigneux, P. Partially segregated neural networks for spatial and contextual memory in 
virtual navigation. Hippocampus, 18:503-518. (2008).

40. Stawarczyk, D., Bezdek, M.A., \& Zacks, J.M. Event representations and predictive processing: The role of the midline default network core. Topics in Cognitive Science. (2019).

41. Smallwood, J., \& Schooler, J. The restless mind. Psychological Bulletin, 132, 964-958. (2006).

42. Baror, S., Aminoff, E. and Bar, M. Proactive by Default. In Ochsner, K. and Gilead, M. (Eds) The Neural Bases of Mentalizing. Springer Press. (in press).

43. Harel, I.I.A. Groen, D.J. Kravitz, L.Y. Deouell, \& C.I. Baker. The temporal dynamics of scene processing: a multifaceted EEG investigation. eNeuro, 3, 1-18. (2016).

44. Bastin, J., Committeri, G., Kahane, P., Galati, G., Minotti, L., Lachaux, J. P., \& Berthoz, A. (2013). Timing of posterior parahippocampal gyrus activity reveals multiple scene processing stages. Human Brain Mapping, 34(6), 1357-1370.

45. Kveraga, K., Ghuman, A. S., Kassam, K. S., Aminoff, E., Hämäläinen, M.S., Chaumon, M., \& Bar, M. Early onset of neural synchronization in the contextual associations network. Proceedings of the National Academy of Sciences of the United States of America, 108(8), 3389-3394. (2011).

46. Võ, M. L. H., \& Wolfe, J. M. Differential Electrophysiological Signatures of Semantic and Syntactic Scene Processing. Psychological Science, 24(9), 1816-1823. (2013).

47. Dorsch, F. Focused daydreaming and mind-wandering. Review of Philosophy and Psychology, 6, 791-813. (2015).

48. Pelagatti, C., Binda, P., \& Vannucci, M. A closer look at the time course of mind wandering: Pupillary responses and behavior. PLoS ONE, 15, (4): e0226792. (2020). 
49. Compton, R.J., Gearinger, D., \& Wild, H. The wandering mind oscillates: EEG alpha power is enhanced during moments of mind-wandering. Cognitive, Affective, \& Behavioral Neuroscience, 18(5), 1-8. (2019).

50. Knyazev, G., Slobodskoj-Plusnin, J., Bocharov, A., \& Pylkova, L. The default mode network and EEG alpha oscillations: An independent component analysis. Brain Research, 1402, 67-79. (2011).

51. Visscher, K.M., Miezin, F.M., Kelly, J.E., Buckner, R.L., Donaldson, D.I., McAvoy, M.P., Bhalodia, V.M., Petersen, S.E., Mixed blocked/event-related designs separate transient and sustained activity in fMRI. Neuroimage, 19, 1694-1708. (2003).

52. Petersen, S.E., \& Dubis, J.W. The mixed block/event-related design. NeuroImage, 62(2), 1177-1184. (2012).

Acknowledgments: We thank Edona Gjonbalaj for her help in collecting the data. This work was supported by Fordham University.

Author Contributions: S.B. and E.A. designed the experiments with input from M.B. S.B.

collected and analyzed the data. S. B. and E.A. interpreted the results. S.B., E.A., \& M.B. wrote the manuscript. All authors approved the final version of the manuscript.

The authors declare no competing interests. 